\title{
An Online Misbehavior Detection Model for Intelligent Transportation Systems
}

\begin{abstract}
AUTHOR
Sultan Ahmed Almalki,

mputer Science Department University of Idaho Moscow, USA

Frederick Sheldon,

Computer Science Depatment University of Idaho Moscow, USA

\section{ABSTRACT}

Cooperative Intelligent Transportation Systems (clTSs) is one of the Internet of Things (OT) applications whose purpose is to improve road safety and traffic efficiency. Within this system, vehicles can communicate with one another by establishing Vehicular Ad-Hoc Network (VANET) along the road section. Although such connectivity facilitates the exchange of information related to road safety and traffic efficiency, it puts the vehicles at isk in that an attacker could compromise one or more vehicles and use them to share false information causing congestions and/or life-threatenin accidents. Although several studies tried to address this issue, they assume that the network topology and/or attack behavior is stationary, which is not realistic as the cITS is dynamic in nature and the attackers may have the ability and resources to change their behavior continuously. Therefore, these assumptions are not suitable and lead to low detection accuracy and high false alarms. To this end, this paper proposes a misbehavior detection model that can cope with the dynamicity of both cITS topology

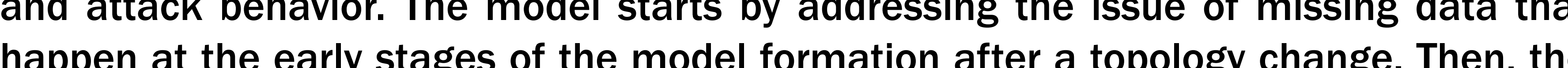
the discriminative features used to train. the expect that the proposed model will help to overcome the limitations of nel Wed solutions by detecting attacks that change their behavior continuously.
\end{abstract}

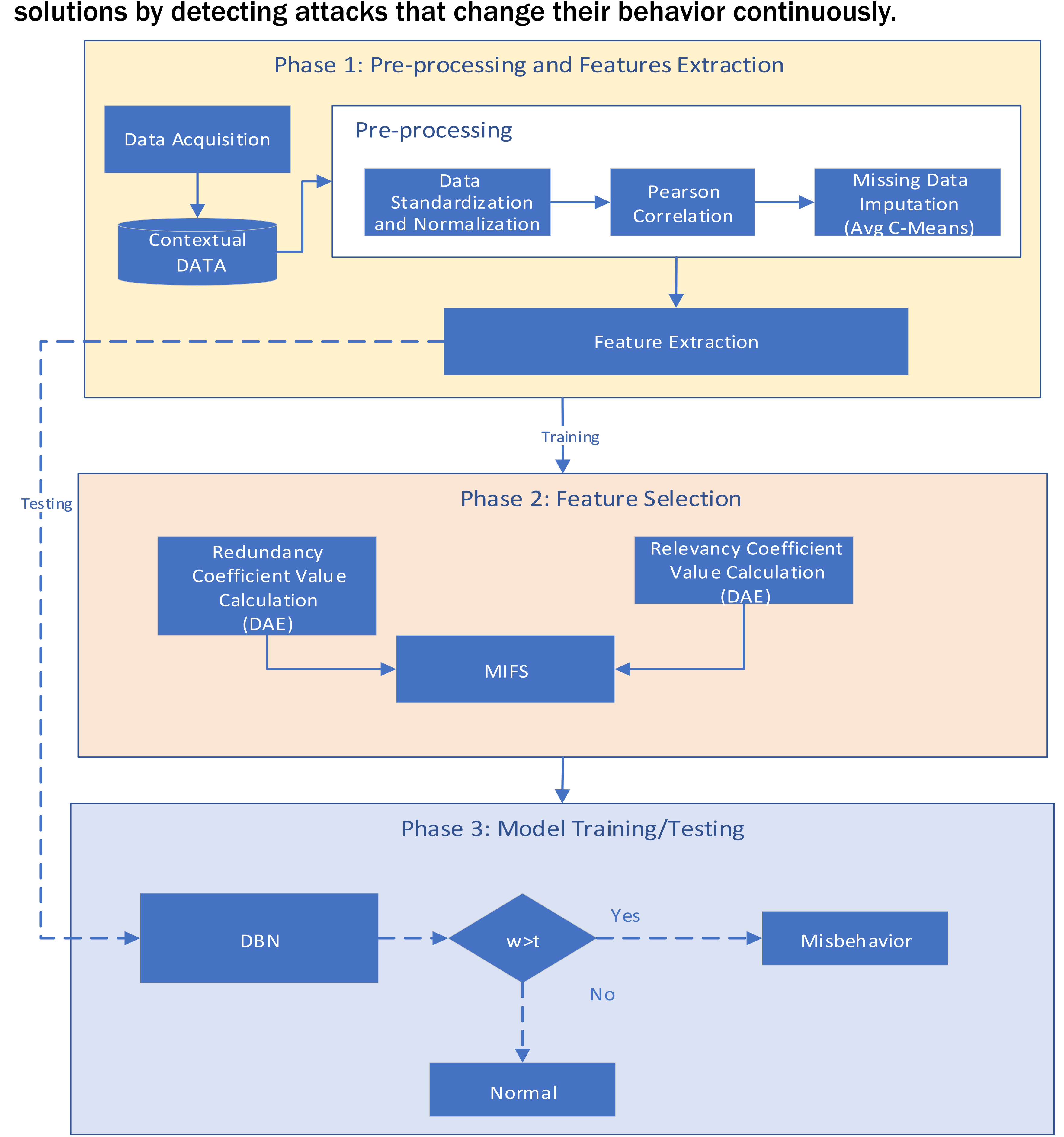

\section{Contribution}

A correlation-based averaged fuzzy C-Means data imputation technique that estimates the missing values in the data collected at the early stages of online model's formation based on the local(univariate) and global(multivariate) average.

A Deep Mutual Information Feature Selection (DMIFS) which incorporates the Deep Autoencoder (DAE) to calculate the values of redundancy and relevancy coefficients accurately when selecting the informative features from the dataset collected at the early stages of online model's formation.

Semantical features will be extracted and fed into the detection model in order to cope with the dynamic nature of the attacks behavior to improve detection polymorphic attacks and readjust the security parameters accordingly.

\section{PROPOSED MODEL}

Three phases are involved in the design and implementation of the model i.e. Pre-processing and Feature extraction, Feature Selection, and model training/testing.

1. During the first phase, the data undergo several pre-processing steps including the standardization, normalization and missing values imputation. The outcome of this phase is the contextual data without missing values.

2. During the 2 nd phase, feature selection process is conducted to build the semantical features set based on the contextual information exchanged between the cITS nodes. The outcome of this phase is the contextual features extraction technique.

3. At the 3rd phase, a deep learning-based detection model will be built by training Deep Belief Network algorithm with the semantical features set extracted during the phase 2 . The outcome of this phase will be the semantic-aware MDS mode

\section{Dataset}

The dataset in this research will be the Next Generation Simulation (NGSIM) Vehicle Trajectories Dataset. It is an open source and publicly available data set with a collection of real-world vehicles' trajectories collected by smart vehicles and consist of different patterns representing different dive situations and driver behavior. Particularly NGSIM was built by collecting data fom vection with $500 \mathrm{~m}$ long and seven lane highway. For each vehicle, the data is collected (recorded) for 45 minutes using 16 sensors .

\section{EVALUATION METRICS}

To evaluate the performance of the proposed MDS for cITS, this study used the accuracy, precision, F-measure as they are common metrics widely used by the extant research. In addition, the approximation error of the proposed MDS model is measured by false positive rates and false negative rates. These measures are well -known and commonly used by the extant research as well. Below equations are used to calculate the detection accuracy, detection rate, precision, false positive rate and the $\mathrm{F}$ measure, respectively; where $T P, T N, F P, F N$ denote the true positive, true negative, false positive and false negative respectively.

$$
\begin{gathered}
A C C=\frac{T P+T N}{T P+T N+F P+F N} \\
D R=\frac{T P}{T P+F N} \\
\text { Precision }=\frac{T P}{T P+F P} \\
F P R=\frac{F P}{F P+T N} \\
F 1=\frac{2 \times \text { Precision } \times \text { Recall }}{\text { Precision }+ \text { Recall }}
\end{gathered}
$$

Conclusion: In this paper, a misbehavior detection model is proposed that address the limitation of existing solutions that assume that the nature of cITS topology as well as the attack strategy are stationary. The model consists of three main components, pre-processing, feature selection, and training/testing. The pre-processing estimates the missing data that occur at the early stages of model formulation after the change occurs. The discriminative features are chosen at the feature selection stage by employing a deep auto encoder to calculate the redunclancy coefficients (2) morel by training the DBN uning the data and features prepared and selected during the 1 st and 2nd phases. In our future work, the three components of the model will be developed and integrated into an MDS model that can prevent evasive attacks that target the cITS ecosystem and protect the data exchanged between vehicles from being manipulated, jammed and/or spoofed.

\section{Universityof Idaho}

